\title{
Theory of Sampling-an approach to representativity offering front line companies added value and potential substantial savings
}

Fritz Rendeman, ${ }^{a}$ Jorgen Riis Pedersen ${ }^{b}$ and Kim H. Esbensen ${ }^{c}$

aScion-DTU, Diplomvej 373, Ø2, 2800 Kgs. Lyngby, Denmark. fritz@rendemann.dk, www.intoscandinavia.com, www.rendemann.dk

bBusiness Consultant, Sense Leadership Coaching. jiiispedersen@gmail.com

'Owner, chief consultant, independent researcher at KHE Consulting, Aldersrogade 8, Copenhagen DK-2100, Denmark; guest-, associate-, visiting professor (Denmark, Norway, Puerto Rico, Quebec). khe.consult@gmail.com

Previous Sampling Columns have dominantly focused on the technical issues of representative sampling. This column addresses sampling from the complementary point of view: "What is the economic and commercial impact from nonrepresentative sampling on management decisions and in boardrooms?" We have invited two experienced business consultants to help scope out an outline indicating powerful opportunities for added value and for substantial savings.

\section{In medias res}

Sampling is the process of selecting and extracting a small part of material suitable for analysis under the critical demand that it is a guaranteed representation of the much larger original lot.

A lot is the sampling target material residing in, for example a shipload, a railroad carriage, a truckload... or constituting a process flow, a moving stream of matter.

Sampling is a technical operation influencing the validity of decision making: sampling affects the bottom line in major sectors of world trade...

Sampling lies behind a significant number of claims and disputes related to commercial transactions...

Sampling leads to a fundamental uncertainty about material, product or goods characterisation, which, if ignored, may translate into economic consequences in the form of hidden value losses...

The principal reason for the above uncertainties is material heterogeneity.
There are huge adverse risks associated with heterogeneous materials, which must be sampled according to codified procedures that specifically counteract heterogeneity in appropriate ways.

Sampling must always be carried out in a representative fashion, by a competent legal person, i.e. a properly trained sampling technician, process engineer, supervisor, or a certified department, institution or agency.

Sampling matters greatly-from the point of view of providing a reliable
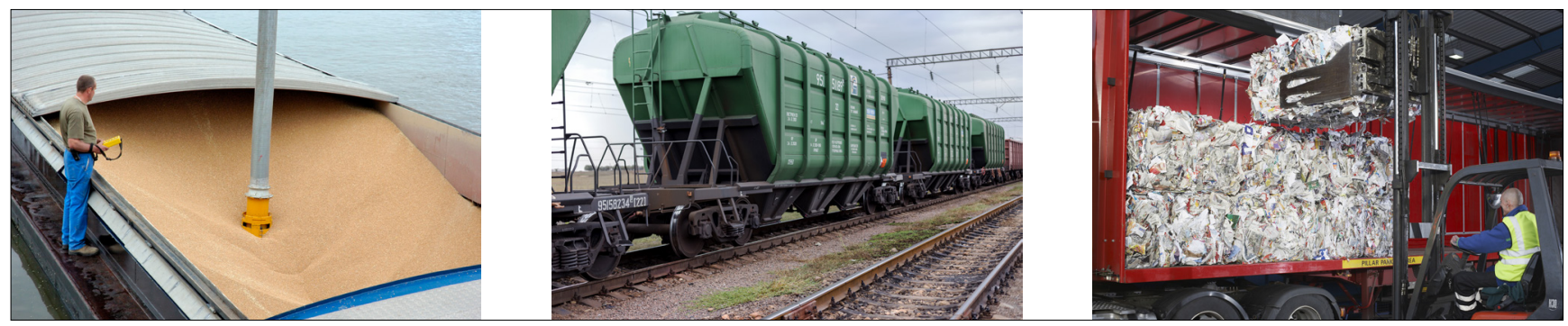

Figure 1. Sampling targets (lots) come in a great variety of shape, form and size, and apparently with no common traits. However, the TOS stipulates how it is only necessary to take their degree of heterogeneity into account. 
basis for decision making in all of science, technology, industry and society. Without a minimum of proper knowledge, competence and experience, there is no guarantee that a "repeated sampling operation" will result in a "duplicate sample" with the same analytical result, precisely because of material heterogeneity.

It is only the specific sampling process with which a sample was extracted that can be designated as representative, or not, according to certain criteria which are codified in the Theory of Sampling (TOS). Material extracted without a documented TOS-basis can never be designated as representative samples-only as worthless small lumps of matter without a meaningful provenance w.r.t. the original lot. Such non-representative extracts, "specimens", must be discriminated against and never relied upon. Specimens are not worth processing in the analytical laboratory, and far less analysed, as their analytical results will be fraught with uncontrollable uncertainties of quite unacceptable magnitudes. Sampling uncertainties are 10-25-50 times larger than the traditional analytical uncertainties which are usually the only determinants in contractual specifications. This misunderstanding is the source of many contractual disputes, but which are wholly avoidable and therefore unnecessary. The TOS is the only competence basis with which to address all these issues. ${ }^{1-4}$

\section{Impact on critical decision making}

This issue is not always fully recognised and acknowledged, and may not always be passed on to all decision making levels, CEO, boardroom. Yet all decisions with critical economic consequences are made here. If there is a fundamental lack of understanding of the magnitudes of such "hidden" uncertainties, there is a lack of due diligence by those responsible for producing correct and reliable documentation upon which to base management decisions on the operative level, or strategic deliberations at the company board room level. This is why there must be at least a minimum core understanding of such "technical sampling issues" at management and board room level as well.

In a commercial transaction, sampling produces the basis for a reference to an agreed product specification, or acts as a quality reference both at pre-shipment inspections as well upon arrival control at the destination. Documented samples are often used as reference material and deposited at the Chamber of Commerce for use in case of legal dispute. Furthermore, if payments of goods are conditioned on a "Letter of Credit" transaction, the sampled reference material (with its certified analytical result) is the decisive factor for payment approval and for the go-ahead process of the shipment in question.

But what if the supposed representative samples are, in fact, worthless specimens?

The contractual apparatus can then be fundamentally undermined with easily imaginable adverse consequences. Sampling matters greatly-at all levels in any company, corporation and organisation.

\section{Sampling-minimum technical knowledge}

All materials are heterogeneous on one scale or another, it is only a matter of degree. Sample representativity is the imperative criterion that must be honoured in order to draw valid conclusions about the true characteristics of an original lot, of materials, of material processes. The force of the TOS becomes clear when it is realised that the TOS is applicable to all types of material; exactly the same principles and practical sampling rules need to be invoked.

Internally in a company, choosing and using only correct sampling procedures, and knowing how to choose only correctly designed equipment, is a critical facility for being able to reach a desired Quality Control/Quality Assurance (QC/ $\mathrm{QA}$ ) level. Using only representative sampling allows a company to optimise the quality of the processing taking place, or of the quality of the products produced. There is a plethora of "hidden" sampling necessary to be able to document QC/QA appropriately. Are all companies or other stakeholders fully

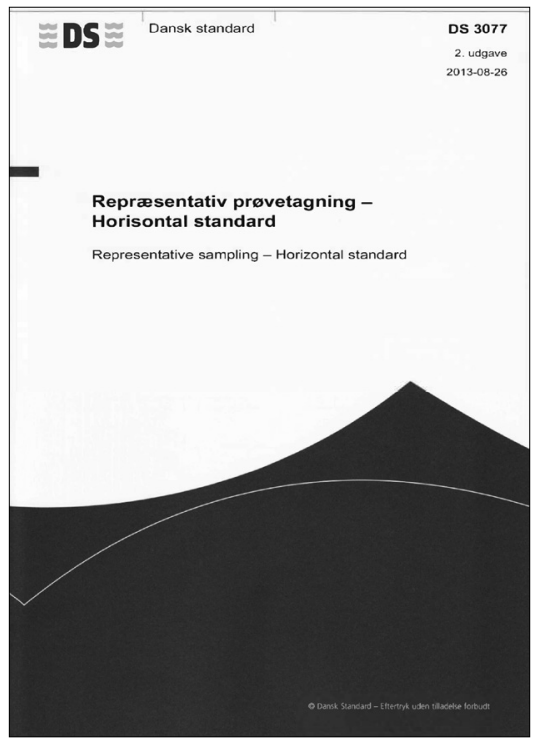

Figure 2. DS3077 (2013): the de facto international standard for representative sampling.

equipped for this task? The TOS is a critical success factor for proper monitoring of quality.

Proper sampling and laboratory analysis become critically important competences. There has been a growing historical usage of proper sampling concepts, methods and equipment over the last 50 years, but even today this is far from universally applied. This historical development is fully documented, indeed available at all levels of appreciation. $^{1-4}$ First and foremost, there is documentation in the form of a de facto international standard devoted to the general principles, equipment design characteristics and, perhaps most important, the necessary and sufficient rules for practical representative sampling based exclusively on the TOS.

\section{Summing up—cardinal points}

Analysing a non-representative specimen is pointless! If a sample cannot be documented to be representative of the lot/ target material from where it was taken, it is a waste of time, effort and money to analyse it.

Erroneous sampling is, every year, responsible for:

waste in production,

- sub-optimised product quality levels, 


\section{Introduction to the Theory and Practice of Sampling}

Kim H. Esbensen

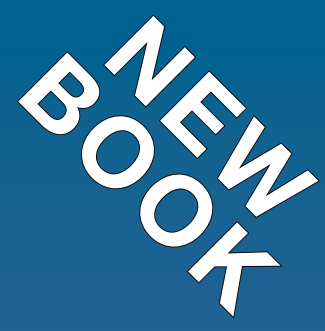

with contributions from Claas Wagner, Pentti Minkkinen, Claudia Paoletti, Karin Engström, Martin Lischka and Jørgen Rils Pedersen

"Sampling is not gambling". Analytical results forming the basis for decision making in science, technology, industry and society must be relevant, valid and reliable. However, analytical results cannot be detached from the specific conditions under which they originated. Sampling comes to the fore as a critical success factor before analysis, which should only be made on documented representative samples. There is a complex and challenging pathway from heterogeneous materials in "lots" such as satchels, bags, drums, vessels, truck loads, railroad cars, shiploads, stockpiles (in the kg-ton range) to the miniscule laboratory aliquot (in the $g-\mu \mathrm{g}$ range), which is what is actually analysed.

This book presents the Theory and Practice of Sampling (TOS) starting from level zero in a novel didactic framework without excessive mathematics and statistics. The book covers sampling from stationary lots, from moving, dynamic lots (process sampling) and has a vital focus on sampling in the analytical laboratory.

"I recommend this book to all newcomers to TOS" "This book may well end up being the standard introduction sourcebook for representative sampling."

"One of the book's major advantages is the lavish use of carefully designed didactic diagrams"
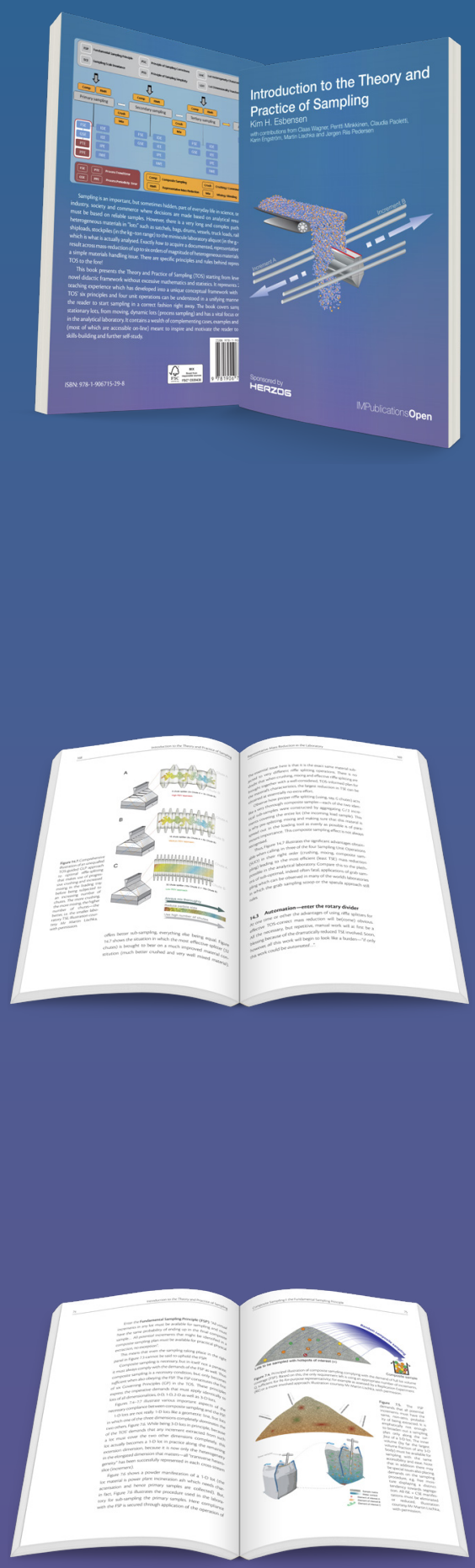
$\square$ erroneous QC/QA documentation, a volume of unwanted complaints, claims, legal disputes and lawsuits, but all this is largely unnecessary! Non-representative sampling also results in damage to companies' reputations and may, at worst, translate into lost business opportunities.

Sampling, although strictly a technical operation, nevertheless deserves the full attention of management. Ignorance of the TOS results in unacceptably high risks in decision making. If a "sample" does not qualify as representative (in reality a "specimen"), it may be part of the reason for significant production loss, commercial disputes, disruption of a commercial partnerships and, potentially, high costs for third parties too.

The importance of sampling must be understood and acknowledged by all stakeholders. The person, department or other entity responsible for sampling must possess a minimum of relevant TOS competence, practical know-how, proper training and integrity, so as professionally to be able to fulfil this vital role in reaching the quality objectives and contractual obligations committed to at higher levels in a company or corporation. It is necessary to establish an entity with a unified responsibility for sampling "from-lot-to-analysis", which has undisputed carte blanche to perform

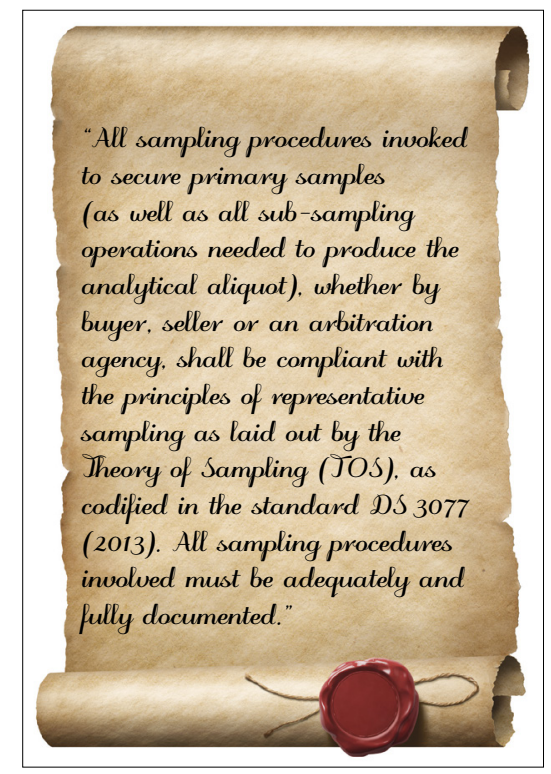

Figure 3. Example of a generic text that would eliminate all sampling vs analysis uncertainties in commercial contracts.

the necessary QC/QA of all sampling processes throughout and across all department borders.

Globalisation and increased volumes of international trade produce a need for relevant rules and regulations. The scenarios depicted here will be the same for all international economic activity levels. From pre-corona prosperity to post-corona recovering economies, all technical and business issues are identical. Therefore, standards and certification bodies have established common guidelines and legal references for cross border transactions and safety regulations.

What has been missing up to now, the missing link, is one comprehensive, universal standard for sampling: DS3077 (2013) provides this.' A unanimously adopted horizontal standard for representative sampling is the logical guardian, which all stakeholders and parties should be able to agree upon. An example of its deployment in the generic buyer-seller scenario is shown in Figure 3.

\section{References}

1. DS 3077. Representative SamplingHorizontal Standard. Danish Standards (2013). http://www.ds.dk

2. K.H. Esbensen, Introduction to the Theory and Practice of Sampling. IM Publications Open, Chichester, UK (2020). impopen.com/sampling

3. F.F. Pitard, The Theory of Sampling and Sampling Practice, $3^{\text {rd }}$ Edn. CRC Press (2019). ISBN: 978-1138476486

4. K. Esbensen and G. Lyman, "'Critique of Gy's Sampling Theory"': Misplaced expectations of Wikipedia's democratic intentions", TOS forum 1, 28-30 (2013). https://doi. org/10.1255/tosf.11

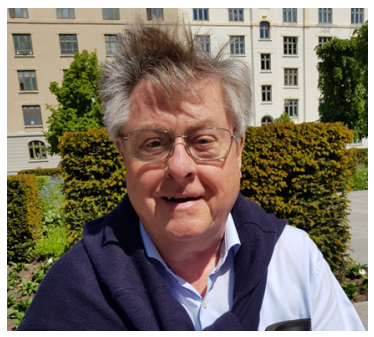

Fritz Rendemann

- MBA, IMI, Geneva, Switzerland

- Business Management

- International Business Development

- Corporate Restructuring \& Turnaround Management

- Strategic Planning

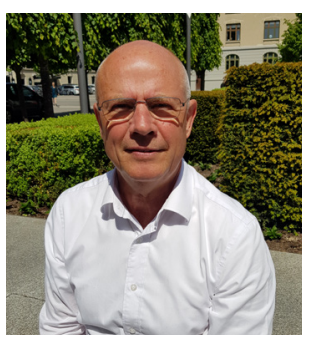

Jørgen Riis Pedersen

- Ph.D. Sociology, EHESS, University of Paris

- Change Management and Strategy

- Corporate Rightsizing

- Organisational Development

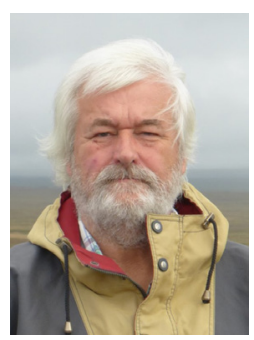

Kim H. Esbensen

M.Sc. University of Aarhus, Denmark - PhD Technical Univ. Denmark (DTH)

- Geology, metallurgy, Data Analysis, Chemometrics

- Theory and Practice of Sampling (TOS)

- Science education outreach 